04

\title{
Исследование лазерной плазмы в среде разреженного газа с использованием одиночного электростатического зонда
}

\author{
(ㄱ С.Г. Давыдов, А.Н. Долгов, А.С. Каторов, В.О. Ревазов, Р.Х. Якубов \\ Всероссийский научно-исследовательский институт автоматики им. Н.Л. Духова, \\ 127055 Москва, Россия \\ I e-mail: katorovalexey@mail.ru
}

Поступило в Редакцию 23 октября 2019 г.

В окончательной редакции 23 октября 2019 г.

Принято к публикации 2 декабря 2019 г.

Исследованы процессы, сопровождающие воздействие на металлическую мишень лазерного излучения наносекундной длительности интенсивностью порядка $10^{13} \mathrm{~W} / \mathrm{m}^{2}$ в вакууме. В качестве средства корпускулярной диагностики был использован одиночный электростатический зонд.

Ключевые слова: зондовая диагностика, лазерная плазма, одиночный зонд, вакуум.

DOI: $10.21883 /$ JTF.2020.06.49277.350-19

\section{Введение}

С момента разработки первых лазеров и по сей день проявлялся интерес к процессу образования приповерхностной лазерной плазмы при воздействии лазерного излучения на вещество. Однако исследования в данной области практически изначально разделились на два направления. Одно из них связано с разработкой технологий модификации поверхности твердого тела, в частности, при плавлении и испарении вещества, что не требует высоких интенсивностей падающего на поверхность мишени лазерного излучения (используемые интенсивности излучения обычно не превышают $\left.10^{11}-10^{12} \mathrm{~W} / \mathrm{m}^{2}\right)$. Другое направление связано с получением экстремальных состояний вещества, например, так называемой релятивистской плазмы, и требует, наоборот, чрезвычайно высоких уровней интенсивности падающего на поверхность мишени лазерного излучения (в настоящее время достигнута величина порядка $10^{25} \mathrm{~W} / \mathrm{m}^{2}$ и даже выше).

Для инициирования разрядов типа вакуумной искры с временем задержки порядка $0.1 \cdot 10^{-6} \mathrm{~s}$ и менее, что входит в круг наших интересов [1], достаточным является уровень интенсивности излучения, падающего на мишень, порядка $10^{13} \mathrm{~W} / \mathrm{m}^{2}$. Задача коммутации вакуумных промежутков с помощью лазерной плазмы продолжительное время оставалась за пределами круга интересов подавляющего большинства исследователей. В последнее время ситуация изменилась: во многих лабораториях ведутся работы по разработке надежных и эффективных вакуумных разрядников с лазерным поджигом в интересах развития техники высоких плотностей энергии [2-6].

\section{Методика эксперимента}

Целью настоящей работы является исследование физических процессов, сопровождающих генерацию лазер- ной плазмы в присутствии разреженной газовой среды при уровне интенсивности излучения на мишени порядка $10^{13} \mathrm{~W} / \mathrm{m}^{2}$ и продолжительности облучения в наносекундном диапазоне.

Длина волны падающего на мишень излучения $\lambda=0.53 \mu \mathrm{m}$. Длительность импульса лазерного излучения по основанию $3 \cdot 10^{-8} \mathrm{~s}$. Интенсивность излучения на мишени порядка $10^{13} \mathrm{~W} / \mathrm{m}^{2}$. Плоская мишень, изготовленная из ковара, и цилиндрический электростатический зонд размещались внутри оптически прозрачной вакуумной камеры, откачиваемой до остаточного давления $\sim 10^{-2}-10^{0} \mathrm{~Pa}$. Средством диагностики являлся одиночный зонд Ленгмюра. Угол падения лазерного луча на мишень был близок к нулю (нормальное падение

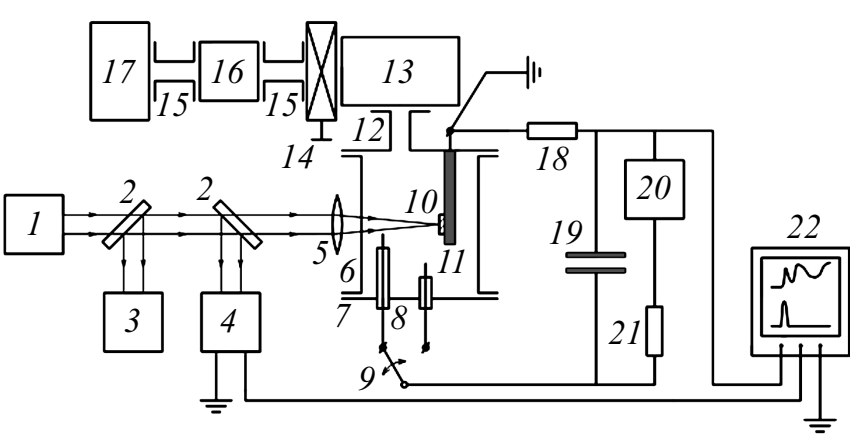

Рис. 1. Схема эксперимента: 1 - лазер ЛТИ-60; 2 полупрозрачное зеркало; 3 - юстировочный Не-Ne-лазер; 4 - быстродействующий фотодатчик; 5 - фокусирующая линза; 6 - оптически прозрачный корпус вакуумной камеры; 7 - фланец; 8 - зонды Ленгмюра; 9 - переключатель; 10 - мишень; 11 - держатель мишени; 12 - фланец (элемент вакуумной арматуры); 13 - магниторазрядный насос; 14 - шибер; 15 - трубопровод; 16 - азотная ловушка; 17 - форвакуумный насос; 18 - низкоиндуктивный резистор сопротивлением $50 \Omega ; 19-$ низкоиндуктивный конденсатор; 20 - источник постоянного напряжения; 21 - зарядное сопротивление $20 \mathrm{k} \Omega ; 22$ - осциллограф. 
на поверхность мишени), а угол между нормалью к поверхности мишени и направлением от пятна фокусировки лазерного луча к зонду составлял $\varphi_{1}=9^{\circ}$ в одном случае и $\varphi_{2}=61^{\circ}$ в другом случае. В первом случае лазерное излучение фокусировалось на мишень на расстоянии от зонда $L_{1}=6 \cdot 10^{-3} \mathrm{~m}$ и во втором случае на расстоянии $L_{2}=12 \cdot 10^{-3} \mathrm{~m}$. Мишень находилась под постоянным нулевым потенциалом. Напряжение, называемое в дальнейшем сигналом с зонда, на согласующем сопротивлении номиналом $50 \Omega$, по которому протекал ток, поступающий на зонд, регистрировалось осциллографом при заданном потенциале зонда (рис. 1). Для полученного при различных значениях потенциала зонда набора осциллограмм сигнала с зонда производились временные срезы, т.е. определялись значения тока на зонд в фиксированный относительно начала импульса лазерного излучения момент времени при различных значениях потенциала зонда. В дальнейшем это позволяло построить вольт-амперные характеристики (BAX) зонда в избранные моменты времени после начала облучения мишени излучением лазера. В качестве положительного тока на зонд был принят, как обычно, ток электронов.

\section{Результаты эксперимента}

На осциллограммах сигнала с зонда, полученных при регистрации разлета плазмы в направлении, близком к нормальному по отношению к поверхности мишени $\left(L_{1}=6 \cdot 10^{-3} \mathrm{~m}, \varphi_{1}=9^{\circ}, \sim 10^{-1} \mathrm{~Pa}\right)$, просматриваются три группы заряженных частиц (рис. 2, цифры в квадратах): I группа возникает практически синхронно с импульсом лазерного излучения; II группа фиксируется примерно через $1.5 \cdot 10^{-7} \mathrm{~s}$ (пик сигнала с зонда, т.е. максимум потока частиц на зонд) после старта лазерного излучения; III группа фиксируется примерно

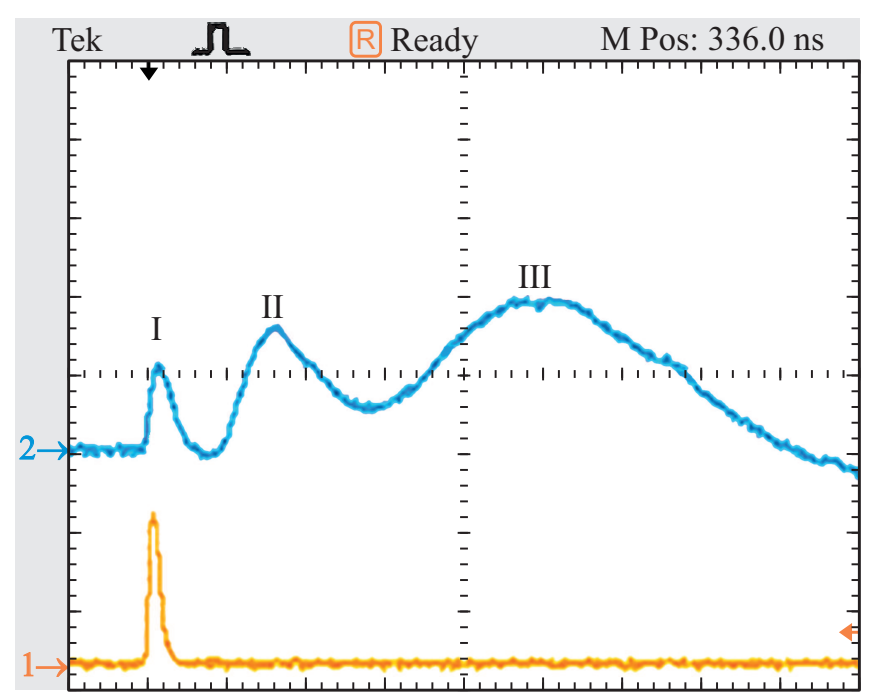

Рис. 2. Осциллограмма сигнала с зонда. Луч $1-$ интенсивность лазерного излучения, луч 2 - ток на зонд, чувствительность $10 \mathrm{~mA}$, развертка $0.1 \cdot 10^{-6} \mathrm{~s} / \mathrm{div}$.

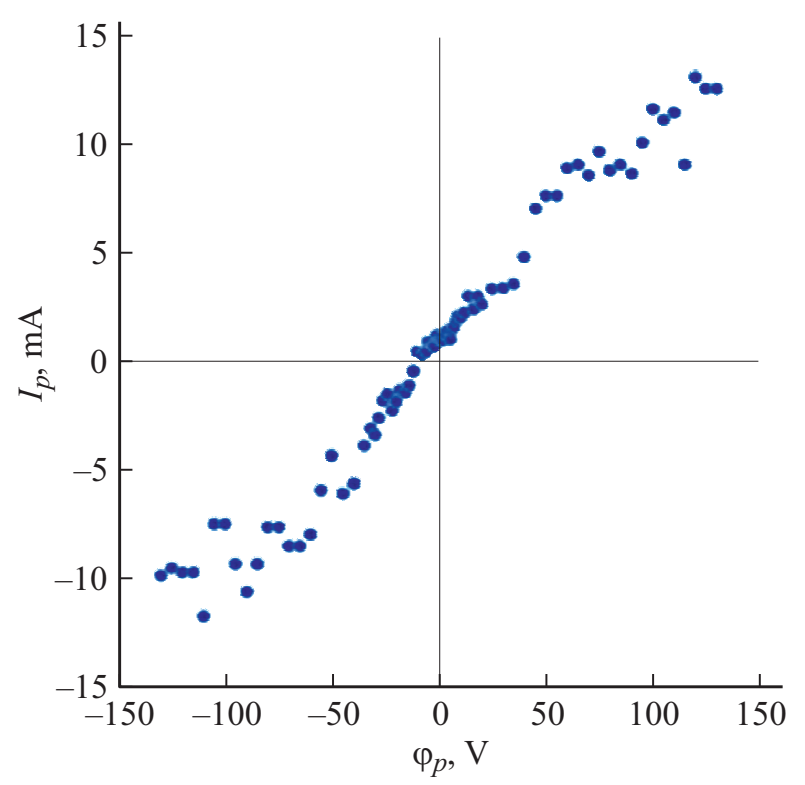

Рис. 3. ВАХ лазерной плазмы, регистрируемая зондом через $0.1 \cdot 10^{-7} \mathrm{~s}$ после старта воздействия лазерного излучения на мишень.

через $\sim 5 \cdot 10^{-7} \mathrm{~s}$ (пик сигнала с зонда) после старта лазерного излучения.

Вид ВАX для I и II групп частиц показывает возможность проведения с их помощью оценки электронной температуры и электронной концентрации, но не дает возможности осуществить оценку концентрации ионов из-за аномально большой силы ионного тока. На ВАХ, соответствующей II группе частиц, отчетливо прослеживается „ступенька“ на электронной ветви, что говорит о том, что II группа частиц - быстро движущийся со скоростью, превышающей ионно-звуковую в плазме, корпускулярный поток [7] (рис. 3). Для BAX II группы частиц характерно отрицательное значение плавающего потенциала на переднем фронте данной группы частиц, что указывает на преобладание потока электронов в этой области. На ВАX, соответствующей I группе частиц (рис. 4), признаки формирования плазменного потока отсутствуют. ВАX, отвечающая III группе частиц, не позволяет сделать никаких заключений, кроме единственного: в плазме, каковой является, по-видимому, эта группа частиц, происходят сильные колебания, собственное электрическое поле которых превосходит по величине поле, создаваемое в плазме зондом (рис. 5) [8,9].

Для I группы частиц на пике сигнала с зонда получены следующие параметры плазмы: $T_{e}=6 \pm 2 \mathrm{eV}$, $n_{e} \approx 6 \cdot 10^{16} \mathrm{~m}^{-3}$. Для II группы частиц: $T_{e}=5 \pm 1 \mathrm{eV}$, $n_{e} \approx 10^{17} \mathrm{~m}^{-3}$. Радиус Дебая при указанных параметрах составит $10^{-5}-10^{-4} \mathrm{~m}$. Так как характерный размер зонда $\sim 10^{-3} \mathrm{~m}$ и, как будет показано ниже, длина свободного пробега частиц $\sim 1 \mathrm{~m}$, то регистрация частиц зондом происходит в бесстолкновительном режиме с тонким переходным слоем. Таким образом, мы имеем право воспользоваться для интерпретации полученных 


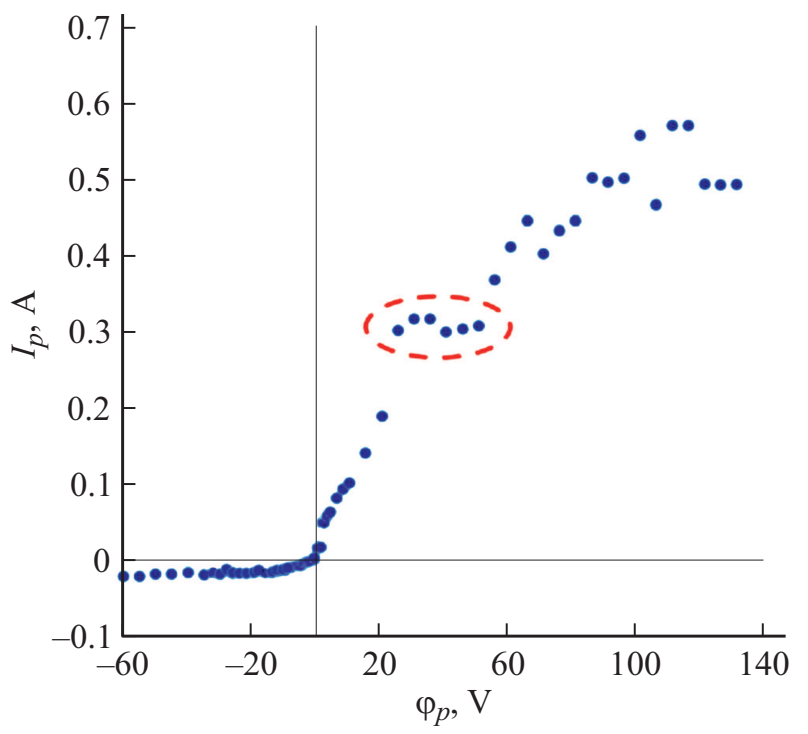

Рис. 4. ВАХ лазерной плазмы, регистрируемая зондом через $1.5 \cdot 10^{-7} \mathrm{~s}$ после старта воздействия лазерного излучения на мишень.

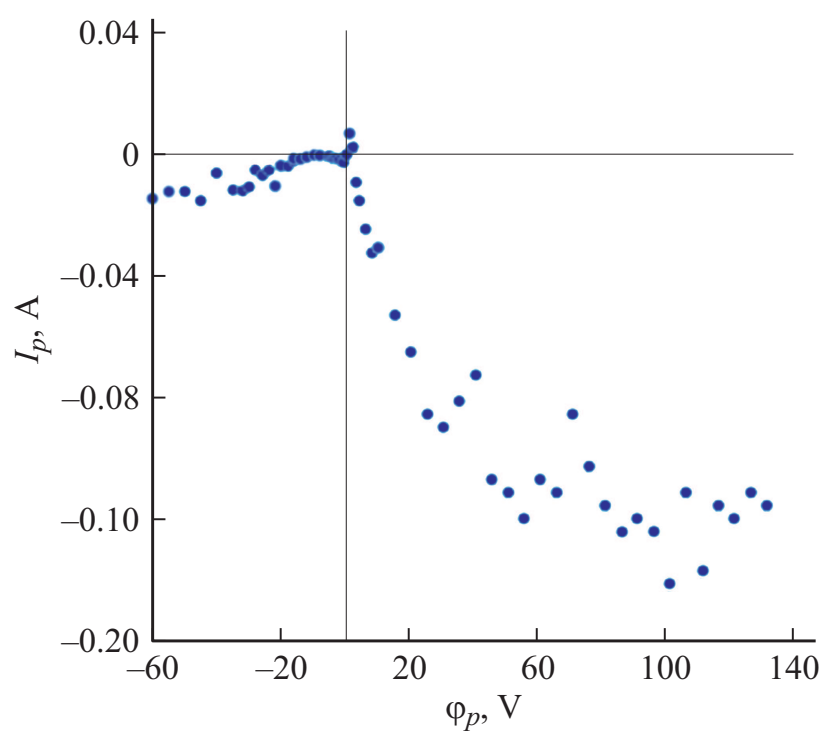

Рис. 5. ВАХ лазерной плазмы, регистрируемая зондом через $3 \cdot 10^{-7}-3.5 \cdot 10^{-7} \mathrm{~s}$ после старта воздействия лазерного излучения на мишень.

результатов классической теорией ленгмюровского зонда, по крайней мере, для переходной области и электронной ветви ВАХ.

Скорости направленного движения частиц во II и III группах, соответствующие максимуму потока и определяемые по времени пролета, составляют примерно $4 \cdot 10^{4}$ и $1.3 \cdot 10^{4} \mathrm{~m} / \mathrm{s}$, а кинетические энергии ионов в плазменном потоке составляют при этом $\sim 500$ и $\sim 60 \mathrm{eV}$ соответственно. Оценка концентрации ионов для указанных групп частиц, исходящая из величины их направленной скорости и учитывающая, что площадь собирающей поверхности зонда в плазменном потоке представляет собой площадь проекции зонда на плоскость, ортогональную направлению потока, дает величины $\sim 3 \cdot 10^{17}$ и $\sim 4 \cdot 10^{18} \mathrm{~m}^{-3}$. Временное разрешение зонда Ленгмюра при полученных значениях температуры и плотности плазмы вполне позволяет производить измерения с временным разрешением в наносекундном диапазоне [7,10].

При регистрации разлета лазерной плазмы в направлении, близком к направлению касательной по отношению поверхности мишени $\left(L_{2}=12 \cdot 10^{-3} \mathrm{~m}, \varphi_{2}=61^{\circ}\right.$, $\left.\sim 10^{-1} \mathrm{~Pa}\right)$, в идентичных условиях проведения эксперимента также просматриваются три группы заряженных частиц. На переднем фронте I группы частиц наблюдается подавляющее преобладание частиц одного знака электронов, вероятно, так называемых убегающих, измеряемая температура которых составляет $T_{e}=48 \pm 6 \mathrm{eV}$, а концентрация оценивается величиной $n_{e} \sim 6 \cdot 10^{17} \mathrm{~m}^{-3}$ (рис. 6). Зафиксированные на пике сигнала с зонда электронные температуры для I и II групп частиц оказались близки к ранее полученным. Кроме того, необходимо отметить, что на электронных ветвях ВАХ в данном случае нет признаков формирования корпускулярного (плазменного) потока в направлении зонда - отсутствует ранее отмечавшаяся „ступенька“. ВАХ, отвечающая III группе частиц, как и в предыдущей серии измерений, отличается аномальным видом, определяемым, по-видимому, развитием в плазме сильных колебаний. Значения скоростей направленного распространения II и III групп частиц, соответствующие максимуму потока и определяемые по времени пролета, сильно размыты и несколько меньше ранее зафиксированных. Регистрируемые концентрации заряженных частиц в I и II группах меньше ранее зафиксированных примерно в $\left(L_{2} / L_{1}\right)^{2}=4$ раз.

При уменьшении давления остаточного газа до $\sim 10^{-2}$ Ра электронная температура на пике сигнала, от-

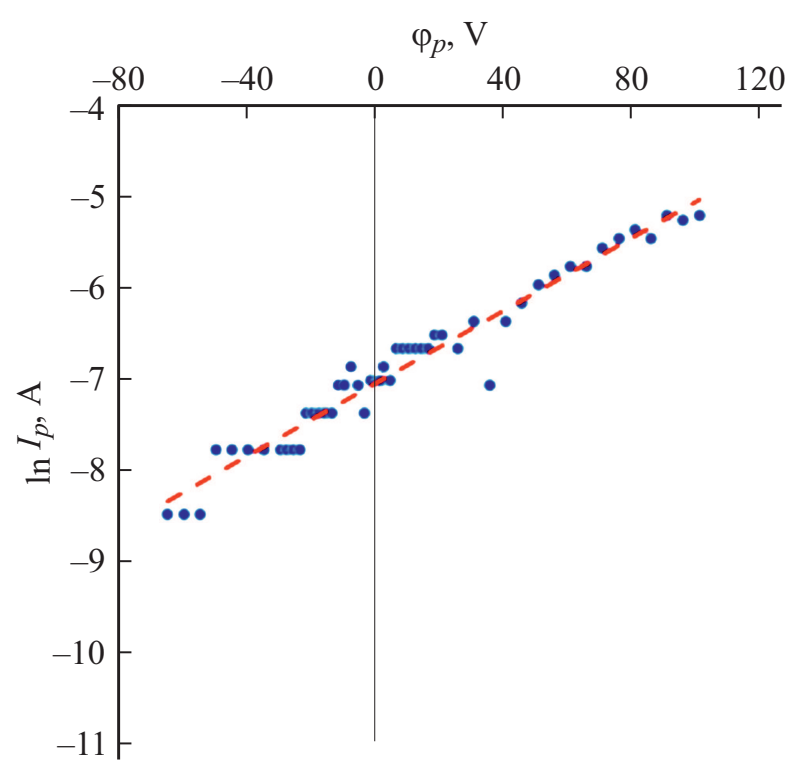

Pис. 6. ВАХ потока электронов. 
вечающего I группе частиц, достигала $20 \pm 4$ и $35 \pm 6 \mathrm{eV}$ для направлений $\varphi_{1}=9^{\circ}$ и $\varphi_{2}=61^{\circ}$ соответственно, а регистрируемые концентрации электронов - падали.

При увеличении давления остаточного газа до $\sim 10^{0}$ Ра наблюдалась только I группа частиц, регистрируемые для обоих направлений электронные температуры составляли $6 \pm 2 \mathrm{eV}$, а концентрации возрастали.

\section{Обсуждение результатов эксперимента}

Разлет лазерной плазмы из области пятна фокусировки происходит недостаточно быстро для того, чтобы обеспечить за время в несколько наносекунд создание вокруг зонда плазменной среды. Появление в спектре ионной эмиссии из лазерной плазмы ионов с энергиями, которые способны обеспечить требуемое экспериментальными данными быстрое появление плазмы в окрестностях зонда, возможно только при достижении несравнимо больших интенсивностей излучения $q \sim 10^{24} \mathrm{~W} / \mathrm{m}^{2}[11]$. Изменения регистрируемой зондом концентрации заряженных частиц I группы коррелируют с изменениями давления остаточного газа, т.е. изначальной концентрацией нейтралов в объеме вакуумной камеры, что свидетельствует в пользу предположения о первоначальном формировании проводящей среды из нейтральных частиц остаточного газа под действием ионизирующего излучения и частиц.

По-видимому, механизм первоначального быстрого образования плазмы связан с воздействием ультрафиолетового излучения и потока быстрых электронов из области пятна фокусировки на остаточный газ в окрестностях зонда. Фиксируемая скорость II группы регистрируемых зондом частиц позволяет предположить, что указанный сгусток плазмы - это результат амбиполярной диффузии в вакуум в результате пространственного разделения заряда ионов и более подвижных электронов [12]. В пользу данного предположения свидетельствует обнаруженное преобладание потока отрицательно заряженных частиц, т.е. электронов, на переднем фронте указанной группы частиц. Наконец, III группа зарегистрированных частиц - это, по-видимому, результат разлета плазмы из области вблизи пятна фокусировки под действием градиента давления электронов [13].

Оценим степень возможного воздействия экспериментально зафиксированного потока электронов с энергиями $\sim 20-50 \mathrm{eV}$ на состояние нейтрального остаточного газа. При концентрации и скорости частиц $\sim 10^{17} \mathrm{~m}^{-3}$ и $\sim 3 \cdot 10^{4} \mathrm{~m} / \mathrm{s}$ соответственно, и длительности существования потока быстрых электронов в течение времени $\sim 10^{-8} \mathrm{~s}$ получим интегральную плотность потока $\Phi_{e} \sim 10^{13} \mathrm{~m}^{-2}$. С учетом сечения взаимодействия $\sigma_{e} \sim 10^{-19} \mathrm{~m}^{2}[14]$ электронов с нейтральными молекулами оценка степени ионизации остаточного газа в окрестностях зонда может составить $\Phi_{e} \sigma_{e} \sim 10^{-6}$, а концентрация заряженных частиц $\sim 10^{12}-10^{14} \mathrm{~m}^{-3}$. При длине свободного пробега электронов $\sim 0.1-10 \mathrm{~m}$ в среде нейтралов и размерах зонда $\sim 10^{-3}$ m ослаблением потока в окрестностях зонда можно пренебречь. В предположении, что ионы в плазме факела имеют небольшие зарядности порядка единицы, и кулоновские столкновения электронов с ионами происходят в условиях максвелловского распределения электронов по скоростям, длину свободного пробега электронов можно оценить с помощью выражения $\lambda=4.5 \cdot 10^{11} \cdot T^{2} /\left(n L_{K}\right)[15]$, где $T$ - электронная температура в Кельвинах, $n$ электронная плотность в $\mathrm{m}^{-3}, L_{K} \approx 15-$ кулоновский логарифм, $\lambda$ измеряется в метрах. Нетрудно убедиться, что длина свободного пробега электронов, обусловленная кулоновским взаимодействием, превышает ранее приведенную величину.

Плазма, образованная путем воздействия импульса лазерного излучения наносекундной длительности и интенсивности $q \sim 10^{12}-10^{14} \mathrm{~W} / \mathrm{m}^{2}$ на твердотельную (или жидкую) мишень, является ярким источником УФ и ВУФ-излучения [16], причем в указанном и актуальном для нас интервале интенсивностей эффективность конверсии энергии лазерного излучения, падающего на мишень, в энергию ВУФ-излучения с энергиями квантов $\sim 10-30 \mathrm{eV}$ составляет примерно от 10 до $80 \%$. Интенсивность $q \approx 3 \cdot 10^{13} \mathrm{~W} / \mathrm{m}^{2}$ достигалась в наших экспериментах при уровне энергии импульса лазерного излучения около $5 \cdot 10^{-3} \mathrm{~J}$. Таким образом, суммарная энергия фотонов, которые способны ионизовать остаточный газ, могла составить порядка $10^{-4} \mathrm{~J}$, а полное число испущенных лазерной плазмой таких фотонов $\sim 10^{14}$. В окрестностях зонда соответственно суммарный поток квантов окажется $\Phi_{f} \sim 10^{18} \mathrm{~m}^{-2}$. При сечении фотоионизации $\sigma_{f} \sim 10^{-21} \mathrm{~m}^{2}$ [17] степень ионизации остаточного газа в окрестностях зонда может составить $\Phi_{f} \sigma_{f} \sim 10^{-3}$, а концентрация заряженных частиц $\sim 10^{15}-10^{17} \mathrm{~m}^{-3}$, что близко к результатам, полученным экспериментально с помощью зондовой методики. Заметим, что длина свободного пробега фотонов в остаточном газе в окрестностях зонда при этом составляет $\sim 10-10^{3} \mathrm{~m}$.

Таким образом, показано, что существуют, по крайней мере, три механизма формирования проводящей среды в окрестностях металлической мишени, находящейся в среде разреженного газа и подвергающейся воздействию импульса оптического излучения наносекундной длительности и интенсивностью порядка $10^{13} \mathrm{~W} / \mathrm{m}^{2}$. Причем наиболее быстрый механизм формирования проводящей среды связан с ионизацией остаточного газа и время задержки момента появления проводящей среды составляет несколько наносекунд относительно момента начала воздействия импульса лазерного излучения на мишень.

Разлет лазерной плазмы происходит с нарушением изотропности. Наиболее быстрое распространение плазмы происходит по нормали к поверхности мишени и носит сверхзвуковой характер. С понижением давления остаточного газа возрастает степень анизотропии разлета лазерной плазмы, что проявляется в первую очередь в заметном увеличении различия регистрируемых 
температур плазмы, разлетающейся в различных направлениях. Обнаружено убегание электронов повышенных энергий, превышающих среднюю регистрируемую тепловую энергию, преимущественно в направлении, близком к касательной к поверхности мишени. Данное обстоятельство можно связать с воздействием на плазму электрического поля волны лазерного излучения.

Тот факт, что с ростом давления остаточного газа перестают регистрироваться две из трех наблюдаемых групп частиц, а точнее сгустков плазмы, можно объяснить следующим образом. Так как в рамках проводимого эксперимента длина свободного пробега заряженных частиц значительно превышает расстояние от пятна фокусировки до зонда, остается предположить, что всему виной коллективное взаимодействие заряженных частиц в плазме. Действительно, II и III группы частиц распространяются в фоновой плазме, образованной в результате ионизации остаточного газа. II группа частиц движется со скоростью, превышающей среднюю тепловую скорость ионов в фоновой плазме, что должно приводить к раскачиванию колебаний в фоновой плазме, рассеиванию частиц на колебаниях и затуханию потока лазерной плазмы [18]. III группа частиц сама представляет собой плазму, в которой происходят сильные колебания, следовательно, будет происходить рассеяние частиц фоновой плазмы на колебаниях, передача энергии от набегающего плазменного потока фоновой плазме и торможение этого потока. С ростом концентрации заряженных частиц в фоновой плазме процесс торможения лазерной плазмы должен протекать более эффективно, хотя бы в силу возрастания массивности фоновой плазмы. Вероятно, в ходе эксперимента мы наблюдали с ростом давления остаточного газа переход через некий рубеж, за которым непосредственное распространение потока лазерной плазмы на расстояние порядка нескольких миллиметров от пятна фокусировки становилось невозможным и приобретало характер диффузии.

Оценим, возможно ли при интенсивности излучения $\sim 10^{13} \mathrm{~W} / \mathrm{m}^{2}$ на мишени, являющейся катодом вакуумного разрядника, непосредственно импульсом излучения инициировать искровой разряд. Указанная выше интенсивность излучения на мишени совпадает с плотностью потока энергии в центре взрывной эмиссии электронов в катодном пятне [12]. Следовательно, плотность эмиссионного тока в пятне фокусировки может достигать величины, характерной для центров взрывной эмиссии электронов $\sim 10^{12} \mathrm{~A} / \mathrm{m}^{2}$. При площади пятна фокусировки $\sim 10^{-7} \mathrm{~m}^{2}$, как это было в нашем эксперименте, возможный эмиссионный ток достигнет величины $\sim 10^{5}$ A. Так как использование вакуумного разрядника с лазерным поджигом выглядит заманчивым, в частности, из-за возможности достижения минимальной индуктивности данного элемента электрической цепи, то примем для дальнейшей оценки расстояние между электродами разрядника равным $\sim 10^{-3} \mathrm{~m}$. Напряжение, которое сможет держать такой зазор в вышеуказанном интервале давлений остаточного газа, можно принять равным $5 \cdot 10^{3} \mathrm{~V}$ [19]. Эффективную площадь поверхности, с которой идет эмиссия электронов, с учетом ионизации остаточного газа примем $\sim 10^{-6}-10^{-4} \mathrm{~m}^{2}$. Величина достижимой силы тока, согласно закону „трех вторых“ $[20]$, составит $\sim 6-600$ А. Средняя скорость ускоряемых в электрическом поле свободных электронов, образовавшихся при ионизации остаточного газа, будет равна $\sim 2 \cdot 10^{7} \mathrm{~m} / \mathrm{s}$. При давлении остаточного газа в несколько суженном интервале $10^{-1}-10^{0} \mathrm{~Pa}$ достижимый ток пучка свободных электронов, который должен дать старт процессу образования центров взрывной электронной эмиссии, составит $\sim 3-30$ А. Таким образом, мы можем ответить положительно на поставленный выше вопрос, т. е. по крайней мере при давлении остаточного газа в диапазоне $10^{-1}-10^{0} \mathrm{~Pa}$ импульсом излучения интенсивностью $\sim 10^{13} \mathrm{~W} / \mathrm{m}^{2}$ на катоде вакуумного разрядника возможно непосредственно, т.е. без промежуточных стадий, например, в виде тлеющего разряда с временем задержки в несколько наносекунд инициировать образование центров взрывной электронной эмиссии и искровой разряд [21] в промежутке протяженностью $\sim 10^{-3} \mathrm{~m}$. Отметим, что использование эффекта оптического пробоя в газе высокого давления потребует интенсивности излучения твердотельного лазера большей на два порядка величины [22].

\section{Заключение}

Исследование физических процессов, сопровождающих генерацию лазерной плазмы в присутствии разреженной газовой среды при уровне интенсивности излучения на мишени порядка $10^{13} \mathrm{~W} / \mathrm{m}^{2}$ и продолжительности облучения в наносекундном диапазоне, дало следующие результаты. При давлении остаточного газа на уровне $10^{-1}-10^{0} \mathrm{~Pa}$ оказалось возможным реализовать быстрый продолжительностью несколько наносекунд механизм образования проводящей среды, связанный в основном с ионизацией остаточного газа излучением лазерной плазмы. Оценки показывают, что подобным образом возможно не только осуществить коммутацию короткого промежутка, но и непосредственно, т.е. без промежуточных стадий, инициировать искровой разряд при начальном напряжении в несколько киловольт.

\section{Конфликт интересов}

Авторы заявляют, что у них нет конфликта интересов.

\section{Список литературы}

[1] Давыдов С.Г., Долгов А.Н., Козловская Т.И., Ревазов О.В., Селезнев В.П., Якубов Р.Х. // Прикладная физика. 2014. № 6. C. 32-35.

[2] Fuukushi I., Watanabe V., Hotta E., Okino A., Ko K.-Ch. // Proc. $19^{\text {th }}$ Int. Symp. on Discharges and Electrical Insulation in Vacuum. Xi'an, 2000. P. 511-514. 
[3] Fei G., Shixiu Ch., Kun Ch. et al. // High Pow. Las. Part. Beam. 2012. Vol. 24. N 4. P. 847-850.

[4] Paperny V.L., Romanov I.V., Korobkin Yu.V., Kiselev N.G. // Proc. XXV Int. Symp. On Discharges and Electrical Insulation in Vacuum. Tomsk, 2012. P. 266-268.

[5] Minfu Liao, Wehao Li, Xiongying Duan, Jiyan Zou, Haoyong Gong. A new type of laser triggered multistage gasvacuum mixed switch // Proc. $2^{\text {nd }}$ Int. Conf. on Electric Power Equipment. Matsue-2013. P. 1-4.

[6] Mao X., He Zh., Wang Y. et al. // IEEE Tr. Plasma Sci. 2014. Vol. 42. N 11. P. 3592-3597.

[7] Козлов О.В. Электрический зонд в плазме. М.: Атомиздат, 1969. $291 \mathrm{c}$.

[8] Чен Ф. Диагностика плазмы / Под ред. Р. Хаддлстоуна и С. Леонарда. М.: Мир, 1967. 515 с.

[9] Nold B., Ribeiro T.T., Ramisch M., Huang Z., Muller H.W., Scott B.D., Stroth U. // New J. Phys. 2012. Vol. 14. P. 063022.

[10] Шотm Л. Методы исследования плазмы / Под ред. В. Лохте-Хольтгревена. М.: Мир, 1971. 552 с.

[11] Ерохин А.А., Кишинеи А.С., Коробкин Ю.В., Романов И.В., Романова В.М., Рупасов А.А., Шиканов А.С. // ЖЭТФ. 2001. Т. 119. Вып. 6. С. 1151-1158.

[12] Месяи, Г.А. Эктоны в вакуумном разряде: пробой, искра, дуга. М.: Мир, 2000. 424 с.

[13] Баренгольи С.А., Месяи Г.А., Шмелев Д.Л. // ЖЭТФ. 2001. Т. 120. Вып. 5(11). С. 1227-1236.

[14] Раховский В.И. Физические основы коммутации электрического тока в вакууме. М.: Наука, 1970. 536 с.

[15] Арцимович Л.А., Сагдеев Р.З. Физика плазмы для физиков. М.: Атомиздат, 1979. $320 \mathrm{c.}$

[16] Амбариумян Р.В., Басов Н.Г., Бойко В.А., Зуев В.С., Крохин О.Н., Крюков П.Г., Сенатский Ю.В., Стойлов Ю.Ю. // ЖЭТФ. 1965. Т. 46. Вып. 6. С. 1583-1587.

[17] Арифов Т.У., Аскарьян Г.А., Тарасова Н.М. // Письма в ЖЭТФ. 1968. Т. 8. Вып. 3. С. 128-132.

[18] Кролл Н., Трайвелпис А. Основы физики плазмы. М.: Мир, 1975. 52 с.

[19] Сливков И.Н. Электроизоляция и разряд в вакууме. М.: Атомиздат, 1972. $304 \mathrm{c}$.

[20] Жданов С.К., Курнаев В.А., Романовский М.К., Цветков И.В. Основы физических процессов в плазме и плазменных установках / Под ред. В.А. Курнаева. М: МИФИ, 2007. $368 \mathrm{c}$.

[21] Месяи Г.А. Взрывная электронная эмиссия. М.: Физматлит, 2011. $280 \mathrm{c}$.

[22] Райзер Ю.П. // Лазерная искра и распространение разрядов. М.: Наука, 1974. 308 с. 